\title{
Positron emission tomography (PET) utilizing Pittsburgh compound B (PIB) for detection of amyloid heart deposits in hereditary transthyretin amyloidosis (ATTR)
}

\author{
Björn Pilebro, ${ }^{\text {a Sandra Arvidsson, }}{ }^{\text {b Per Lindqvist, }}{ }^{\mathrm{b}}$ Torbjörn Sundström, ${ }^{\mathrm{c}}$ \\ Per Westermark, ${ }^{\mathrm{d}}$ Gunnar Antoni, ${ }^{\mathrm{e}}$ Ole Suhr, ${ }^{\mathrm{f}}$ and Jens Sörensen ${ }^{\mathrm{g}}$ \\ a Heart Centre, Cardiology, Department of Public Health and Clinical Medicine, Umeå University, \\ Umeå, Sweden \\ b Heart Centre, Department of Surgical and Perioperative Sciences, Clinical Physiology, Umeå \\ University, Umeå, Sweden \\ c Department of Radiation Sciences, Diagnostic Radiology, Umeå University, Umeå, Sweden \\ d Department of Immunology, Genetics and Pathology, Uppsala University, Uppsala, Sweden \\ e Department of Medicinal Chemistry, Uppsala University, Uppsala, Sweden \\ ${ }^{f}$ Department of Public Health and Clinical Medicine, Umeå University, Umeå, Sweden \\ g Department of Surgical Sciences, Nuclear Medicine \& PET, Uppsala University, Uppsala, \\ Sweden
}

Received Mar 31, 2016; accepted Jul 21, 2016

doi: $10.1007 / \mathrm{s} 12350-016-0638-5$

Background. DPD scintigraphy has been advocated for imaging cardiac amyloid in ATTR amyloidosis. PET utilizing ${ }^{11} \mathrm{C}$-Pittsburgh compound B (PIB) is the gold standard for imaging brain amyloid in Alzheimer's disease. PIB was recently shown to identify cardiac amyloidosis in both AL and ATTR amyloidosis. In the ATTR population, two types of amyloid fibrils exist, one containing fragmented and full-length TTR (type A) and the other only full-length TTR (type B). The aim of this study was to further evaluate PIB-PET in patients with hereditary ATTR amyloidosis.

Methods. Ten patients with biopsy-proven V30M ATTR amyloidosis and discrete or no signs of cardiac involvement were included. Patients were grouped according to TTR-fragmentation. All underwent DPD scintigraphy, echocardiography, and PIB-PET. A left ventricular PIB-retention index (PIB-RI) was established and compared to five normal volunteers.

Results. PIB-RI was increased in all patients $(P<0.001)$, but was significantly higher in type $B$ than in type A $\left(0.129 \pm 0.041\right.$ vs $\left.0.040 \pm 0.006 \mathrm{~min}^{-1}, P=0.009\right)$. Cardiac DPD uptake was elevated in group $A$ and absent in group $B$.

Conclusion. PIB-PET, in contrast to DPD scintigraphy, has the potential to specifically identify cardiac amyloid depositions irrespective of amyloid fibril composition. The heart appears to be a target organ for amyloid deposition in ATTR amyloidosis. (J Nucl Cardiol 2018;25:240-8.)

Key Words: Cardiomyopathy $・$ amyloidosis $・$ Pittsburgh compound B

\footnotetext{
Funding Supported by FAMY, FAMY Norrbotten and Amyl Foundation, Swedish Heart and Lung Foundation, Västerbotten County Council and Selander's foundation.

Reprint requests: Björn Pilebro, Heart Centre, Cardiology, Department of Public Health and Clinical Medicine, Umeå University, 90185 Umeå, Sweden; bjorn.pilebro@umu.se

1071-3581/\$34.00

Copyright $(2) 2016$ The Author(s). This article is published with open access at Springerlink.com
} 


\begin{tabular}{|c|c|}
\hline Abbreviations & \\
\hline ATTR & Transthyretin amyloid protein \\
\hline DPD & $\begin{array}{l}{ }^{99 \mathrm{~m}} \text { Technetium-3,3-diphosphono-1,2- } \\
\text { propanodicarboxylic acid }\end{array}$ \\
\hline IVS & Interventricular septum \\
\hline IVSD & $\begin{array}{l}\text { End-diastolic interventricular septum } \\
\text { thickness }\end{array}$ \\
\hline LA & Left atrium \\
\hline LV & Left ventricle \\
\hline LVEF & Left ventricular ejection fraction \\
\hline${ }^{11} \mathrm{C}-\mathrm{PIB}$ & $\begin{array}{l}\text { Carbon-11 labeled Pittsburgh com- } \\
\text { pound B }\end{array}$ \\
\hline RI & Retention index \\
\hline TTR & Transthyretin \\
\hline
\end{tabular}

\section{BACKGROUND}

Transthyretin (TTR) amyloidosis (ATTR) is a disease where wild-type or mutant TTR assembles into amyloid. It is a systemic disease that affects mainly the heart and nervous system. ${ }^{1}$ Over 100 different diseasecausing mutations in the TTR gene have been identified. ${ }^{2}$ There is a pronounced variation in the presentation of the disease, where neuropathy or cardiomyopathy may dominate, but most mutations have symptoms from both the heart and nervous system. In addition, being a systemic disease, gastrointestinal, renal, visual, and other organ systems can be involved. These phenotypic variations are in part tied to the disease-causing mutation and probably age at onset, but also to others as yet unknown factors. $^{3}$

Proteolysis and fragmentation of TTR appear to play a central role in the development of TTR cardiomyopathy. ${ }^{4,5}$ Among patients with ATTR V30M amyloidosis, the most common TTR mutation in Europe, two phenotypes are noted: one characterized by late-onset disease $(50 \geq$ years of age) and with both cardiac and neurological manifestations, and the other by an early-onset disease ( $<50$ years of age) and with mainly neuropathic manifestation. ${ }^{6}$ These phenotypes have been tied to differences in amyloid fibril composition. ${ }^{4,5}$ Amyloid from patients with late-onset disease contains both full-length and N-terminal TTR fragments starting at position 46-52 (Type A fibril pattern), whereas amyloid of early-onset disease patients typically contains full-length TTR only (Type B fibril pattern). ${ }^{7}$ The type $\mathrm{B}$ fibril pattern has so far only been detected in the $\mathrm{V} 30 \mathrm{M}$ population and in patients carrying the rare Y144C mutation. Amyloid deposits from patients with other mutations or wild-type ATTR amyloidosis have all contained TTR fragments. ${ }^{8}$ Until recently, the only available treatment for ATTR amyloidosis was liver transplantation. However, subsequent analysis has shown that patients with type B fibrils, generally early-onset V30M patients had a substantial better outcome compared with type A patients, i.e., generally late-onset $\mathrm{V} 30 \mathrm{M}$ and nonV30M patients. ${ }^{9}$ If amyloid fibril composition has any impact on the efficacy of pharmacological treatment has so far not been investigated.

There are currently several diagnostic methods available for detection of ATTR cardiomyopathy. Despite the emergence of other modalities, echocardiography remains the basis for diagnosis and follow-up. For the purpose of early detection of ATTR cardiomyopathy, ${ }^{99 m}$ technetium-3,3-diphosphono-1,2-propanodicarboxylic acid (DPD) scintigraphy is by many considered the gold standard. ${ }^{10}$ The exact mechanisms by which DPD interacts with cardiac amyloid deposits are not known, neither is it clear why some patients with $\mathrm{AL}$ amyloidosis display DPD uptake and others do not.

A class of novel tracers, originating from thioflavin $\mathrm{T}$, has been developed for specific amyloid imaging of the brain in Alzheimer's disease using the positron emission tomography (PET) and is currently regarded as the non-invasive gold standard for amyloid detection in the brain. ${ }^{11}$ Carbon-11-labeled Pittsburgh compound B $\left({ }^{11} \mathrm{C}-\mathrm{PIB}\right)$, the original PET amyloid tracer, should in theory bind to beta-pleated sheets in general, even outside the brain. In a small pilot study ${ }^{11} \mathrm{C}$-PIB PET showed a high affinity for cardiac amyloid deposits ${ }^{12}$ in both light chain amyloidosis (AL) and ATTR amyloidosis. This was reproduced by Lee et al in 22 patients with AL amyloidosis. ${ }^{13}$ Another related tracer, 18FFlorbetapir, has similarly been proven to identify cardiac amyloid deposits with high diagnostic sensitivity, ${ }^{14}$ and specific binding to cardiac amyloid has been shown. ${ }^{15}$ No previous study has investigated amyloid PET-tracer uptake in ATTR amyloidosis patients with known amyloid precursor protein; before further evaluation can be done in larger prospective studies, the impact of amyloid fibril composition on tracer retention needs to be investigated.

The aim of this study was to evaluate the impact of amyloid fibril composition on PET/CT using ${ }^{11} \mathrm{C}$-PIB in biopsy-proven ATTRV30M amyloidosis patients, and to compare the outcome with that obtained by DPD scintigraphy.

\section{MATERIAL AND METHOD}

\section{Patients Selection}

Ten patients with genetically and histopathologically diagnosed neuropathic ATTRV30M amyloidosis were 
included in the study. The amyloid fibril type, i.e., type A or B, had been determined in fat pad biopsy for all patients. Biopsies were analyzed regarding fibril type according to a standardized Western blot method. ${ }^{7}$ We chose to include five patients with type A and five patients with type B fibrils in the study. In addition, to enable investigation of the impact of amyloid fibril type, the patients selected had similar echocardiographic findings displaying little or mild signs of cardiomyopathy, and they were of comparable age and had similar disease duration

\section{Healthy Volunteers}

Five healthy subjects without signs or symptoms of cardiac disease were included ( 3 males, 2 females, age range 54-75). This cohort was also used as healthy controls in a previously published study. ${ }^{12}$

\section{Patient Characteristics}

Patients were interviewed and their files were reviewed for information concerning symptoms, disease duration, cardiovascular comorbidities (hypertension, known vascular disease, renal insufficiency), and previous and current treatment for ATTR amyloidosis. The cardiac biomarkers troponin $\mathrm{T}$ and NT-proBNP were analyzed at the clinical chemistry laboratory of Umeå University Hospital, according to routine practice using a Cobas 8000 machine and troponin T hs STAT and proBNP II STAT reagents (Roche Scandinavia, Bromma, Sweden).

\section{PET/CT Procedure}

After low-dose CT scanning, a 25-minutes dynamic emission scan of the heart was started simultaneously with the intravenous bolus injection of ${ }^{11} \mathrm{C}$-PIB $(6 \mathrm{MBq} / \mathrm{kg})$ using a Discovery ST PET/CT scanner (GE Healthcare). Imaging was performed in 3-dimensional mode, yielding 47 transaxial images. All appropriate corrections for scanner normalization, dead time, decay, scatter, randoms, and attenuation were applied. Images were reconstructed into time frames of 29 frames $(12 \times 5,6 \times 10,4 \times 30,2 \times 60,2 \times 120$, and $3 \times 300$ s) using ordered-subset expectation maximization ( 2 iterations, 21 subsets), with the application of a 5-mm Gaussian filter. Images consisted of $128 \times 128$ voxels, with dimensions of $2.34 \times 2.34 \times 3.27 \mathrm{~mm}$, and a spatial resolution of approximately $7 \mathrm{~mm}$.

\section{${ }^{11}$ C-PIB PET Image Analysis}

The analysis was performed using a dedicated software (Carimas V2.6, Turku, Finland) as previously described. ${ }^{16}$ Briefly, the left ventricular wall was outlined semiautomatically and the regional radioactivity from 10 to 15 minutes after injection was calculated. A small volume of interest was positioned centrally in the left ventricular (LV) cavity, and a time-activity curve from 0 to 15 minutes after injection was

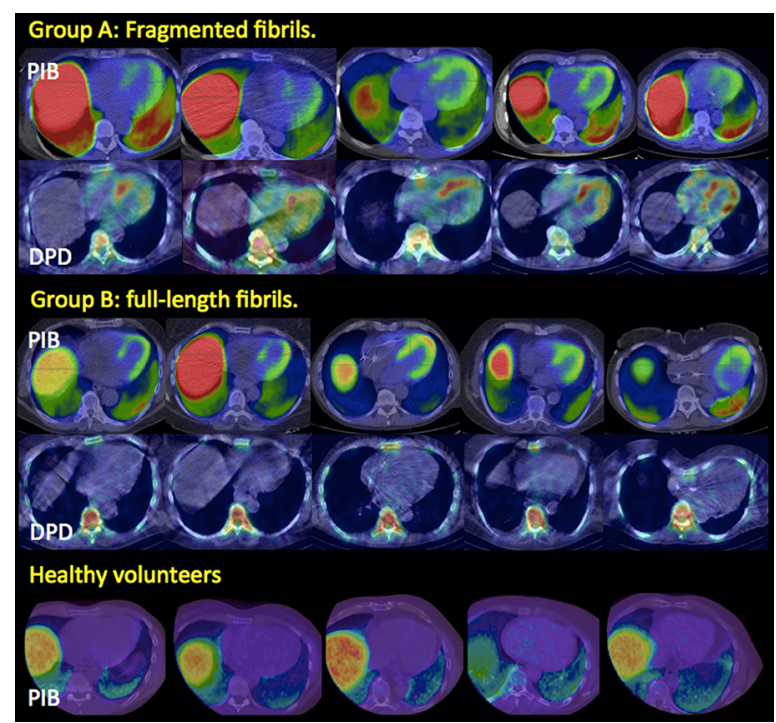

Figure 1. Matched C-PIB-PET (upper) and DPD SPECT (lower) images of patients with type A fibrils (group $A^{11}$ ) and type B fibrils (group B). At the bottom is ${ }^{11} \mathrm{C}$-PIB-PET images from healthy volunteers.

integrated to calculate the area under the curve (AUC). An ${ }^{11} \mathrm{C}$-PIB retention index (RI) was then calculated by dividing LV wall activity by the AUC and global LV RI values. Summed PET images from 15 to 25 minutes after injection were fused with CT to visually confirm cardiac ${ }^{11 \mathrm{C}}$-PIB uptake (Figure 1). The RI was presented as a parametric polar map (see Figure 2)

\section{Echocardiography}

Patients were investigated with two-dimensional and Doppler echocardiography using Vivid E9 (GE Medical systems, Horten, Norway), ${ }^{17,18}$ and offline analysis was carried out using Echopac PC version 113 (GE Healthcare). Enddiastolic interventricular septum (IVS) thickness (IVSD) was measured from parasternal long axis view. From apical fourchamber view, left atrium (LA) volume was measured in endsystole using biplane area-length method and left ventricular ejection fraction (LVEF) was calculated using manual biplane Simpson model. Speckle tracking analysis was performed using automated function imaging (AFI) for calculation of LV global longitudinal strain from apical four-, three-, and twochamber views. The mitral annulus and apex were manually outlined by the use of three index points followed by automatic definition of the endocardial border, mid-layer, and epicardial border in each frame throughout the cardiac cycle. ${ }^{19}$ Reduced $\mathrm{LV}$ global strain was defined as a value $\geq 16 \%$. $^{20}$

\section{DPD Scintigraphy}

All patients were scanned using a hybrid single-photon emission computerized tomography (SPECT)-CT gamma camera (Infinia Hawkeye, GE) with a Low Energy High 


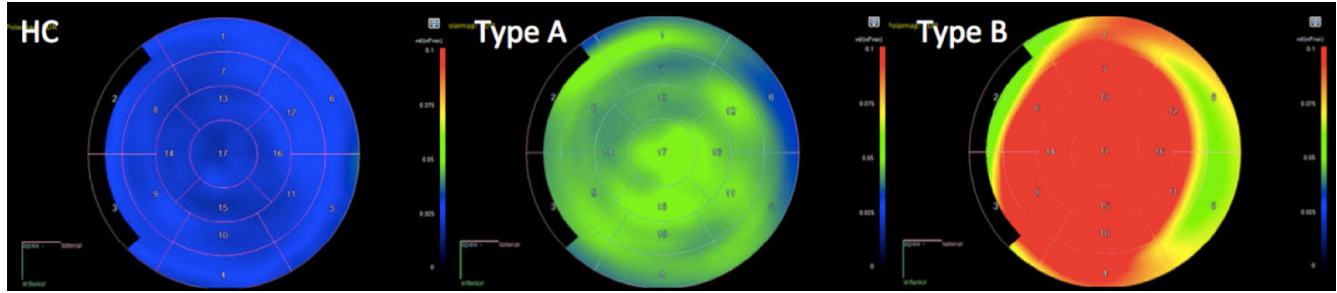

Figure 2. Representative polar maps plots of the ${ }^{11} \mathrm{C}$-PIB retention index in the left ventricular wall of a healthy control subject $(H C)$, a patient with both full-length and fragmented amyloid fibrils (Type A), and a patient with full-length fibrils only (Type B). The images were calibrated to the same absolute color scale with a maximum of $0.1 \mathrm{~mL} \cdot \mathrm{min}^{-1} \cdot \mathrm{mL}^{-1}$.

Resolution collimator after intravenous injection of $740 \mathrm{MBq}$ of DPD. Whole body planar images were acquired 5 minutes and 3 hours postinjection in a $256 \times 1024$ matrix followed by a cardiac SPECT-CT with a low-dose, non-contrast CT scan. SPECT acquisition was made with a $128 \times 128$ matrix size in 30 projections followed by an iterative (OSEM, 3 iterations, 10 subsets) reconstruction with CT-based attenuation correction. SPECT-CT reconstruction and image fusion were performed on a GE Xeleris workstation. The CT volume data were reconstructed into $5 \mathrm{~mm}$ slice thickness.

Visual scoring of cardiac retention was made according to a suggested method ${ }^{11,12}$ (i.e., score 0 , absent cardiac uptake and normal bone uptake; score 1, mild cardiac uptake, inferior to bone uptake; score 2, moderate cardiac uptake accompanied by attenuated bone uptake; score 3 , strong cardiac uptake with mild/absent bone uptake). A score of 1, 2, or 3 was considered as DPD positive. Two experienced specialists in nuclear medicine independently performed image analysis. There were no disagreements.

\section{Endomyocardial Biopsy (EMB)}

To validate the PIB-PET findings in patients with type B amyloid fibril composition, these patients were asked to volunteer to cardiac tissue sampling. One patient had undergone EMB previously and only one of the remaining four agreed to the procedure. Biopsies were taken from the right chamber according to the standard protocol.

\section{Ethics}

The study was conducted according to the Helsinki declaration. All patients had given their written consent. The central ethical review board in Umeå approved all study procedures.

\section{Statistics}

${ }^{11}$ C-PIB RI was compared among Group A and B and Healthy Volunteers using a Friedman test. The group means were compared using Mann-Whitney $U$ tests. Statistical analyses were performed using Graphpad Prism 5.

\section{RESULTS}

\section{Patient Characteristics}

The patient characteristics are summarized in Table 1. There were no significant differences in disease duration, prevalence of hypertension, or age between group A and group B. Three patients with type B fibrils had undergone liver transplant prior to investigationone 16 years prior to, and the other two during the year before the investigation. Three patients were on investigational drug treatment with siRNA (Patirisan, Alnylam). One patient in the type B group had iatrogenic ATTR amyloidosis, having received a liver from a patient with ATTR amyloidosis as part of a domino transplant procedure. ${ }^{21}$ Two patients with type B fibrils had cardiac pacemakers -one due to high level AV-block and the other SA-block; these two patients also hade renal insufficiency and elevated NT-proBNP (Table 2). In one patient with type B fibril composition, endomyocardial biopsy had been performed, which was amyloid positive. None of the patients had undergone cardiac surgery or had known coronary disease. All imaging was performed within one year for each patient.

\section{DPD Scintigraphy}

None of the patients with type B amyloid fibril composition had DPD uptake. Of the patients with type A fibrils, four had grade 2 and one had grade 3 DPD uptake (Table 2; Figure 1).

\section{Echocardiography}

No significant differences were observed between the two patient groups regarding LV dimensions and wall thickness or LA size (Table 3). No patient had increased amounts of pericardial effusion. Seven patients had increased LV wall thickness (wall thickness $>12 \mathrm{~mm}$ ). Patients from both subgroups had mainly LV wall thickening localized to the IVS. One patient 
Table 1. Patient characteristics

\begin{tabular}{|c|c|c|c|c|c|c|}
\hline $\begin{array}{l}\text { Study } \\
\text { ID }\end{array}$ & $\begin{array}{c}\text { Age } \\
\text { (years) }\end{array}$ & Gender & $\begin{array}{c}\text { Disease } \\
\text { duration } \\
\text { (years) }\end{array}$ & $\begin{array}{l}\text { Fibril } \\
\text { type }\end{array}$ & $\begin{array}{l}\text { Primary } \\
\text { symptoms }\end{array}$ & $\begin{array}{l}\text { Medical history } \\
\text { and ATTR treatment }\end{array}$ \\
\hline 1 & 68 & Female & 4 & A & Neuropathy & Hypertension. Patirisan \\
\hline 2 & 71 & Female & 2 & A & Neuropathy & Hypertension \\
\hline 3 & 68 & Male & 1 & B & Neuropathy & Patirisan \\
\hline 4 & 76 & Female & 6 & A & Neuropathy & \\
\hline 5 & 64 & Female & 4 & B & Neuropathy & Liver transplant may 2014 \\
\hline 6 & 66 & Male & 3 & A & Neuropathy & Hypertension \\
\hline 7 & 66 & Male & 11 & B & Renal failure & $\begin{array}{l}\text { Liver transplant December } 2013 \text {, } \\
\text { Hypertension. Renal failure. Cardiac } \\
\text { pacemaker (SA-block) }\end{array}$ \\
\hline 8 & 74 & Male & 2 & B & Neuropathy & latrogenic ATTR amyloidosis ${ }^{a}$ \\
\hline 9 & 65 & Female & 22 & B & Neuropathy & $\begin{array}{l}\text { Liver transplant September } 1997 \text {, } \\
\text { hypertension. Renal failure. Chronic } \\
\text { atrial fibrillation. Cardiac } \\
\text { pacemaker (AV-block) }\end{array}$ \\
\hline 10 & 69 & Male & 4 & A & Neuropathy & Patirisan \\
\hline
\end{tabular}

Fibril type A consists of a mix of full-length and truncated ATTR and type B consists of only full-length ATTR. Disease duration is reported as described by the patients. Primary symptoms are the symptoms leading to health care contact

a Patient 8 had received a liver from a patient with ATTR amyloidosis 14 years prior to the study as part of a "domino transplant procedure"

Table 2. Results

\begin{tabular}{lcccccc}
\hline $\begin{array}{c}\text { Patient } \\
\text { ID }\end{array}$ & $\begin{array}{c}\text { Fibril } \\
\text { type }\end{array}$ & DPD & $\begin{array}{c}\text { IVSD } \\
(\mathbf{m m})\end{array}$ & $\begin{array}{c}\text { LV global strain (normal/ } \\
\text { reduced) }\end{array}$ & $\begin{array}{c}\text { NT-proBNP } \\
\left(\mathbf{n g} \mathbf{L}^{-1}\right)\end{array}$ & $\begin{array}{c}\mathbf{1}^{\mathbf{1}} \mathbf{C}-\mathbf{P I B} \\
\text { RI }\end{array}$ \\
\hline 1 & $\mathrm{~A}$ & 2 & 16 & Normal & 328 & 0.044 \\
2 & $\mathrm{~A}$ & 2 & 12 & Normal & 199 & 0.032 \\
3 & $\mathrm{~A}$ & 0 & 14 & Normal & 134 & 0.138 \\
4 & $\mathrm{~B}$ & 2 & 13 & Normal & 220 & 0.042 \\
5 & $\mathrm{~A}$ & 2 & Normal & 144 & 0.091 \\
6 & $\mathrm{~B}$ & 0 & 11 & Reduced & 134 & 0.046 \\
7 & $\mathrm{~A}$ & 3 & 13 & Reduced & $3256^{\mathrm{a}}$ & 0.179 \\
8 & $\mathrm{~B}$ & 0 & 16 & Normal & 711 & 0.083 \\
9 & $\mathrm{~B}$ & 0 & 12 & Reduced & $2927^{\mathrm{a}}$ & 0.154 \\
10 & $\mathrm{~B}$ & 0 & 11 & Normal & 146 & 0.035 \\
\hline
\end{tabular}

$D P D$ DPD Uptake presented as graded uptake according to Puille et al $^{14}$; IVSD, end-diastolic interventricular septum thickness; ${ }^{11}$ C-PIB RI, PIB retention index

Type A fibrils contain a mix of full-length and truncated ATTR and type B fibrils contains full-length fibrils only

apatient with kidney failure

(number 9) had predominant apical thickening. In two patients, increased LV thickness was localized to the basal part of IVS. Three patients (no 6, 7 and 9) had reduced LV global longitudinal strain. Reduced strain was primarily seen in segments with more pronounced wall thickening.

\section{${ }^{11}$ C-PIB}

All patients, regardless of fibril type, had increased myocardial PIB uptake compared to healthy volunteers $(P<0.001)$ (Figure 1$)$. When measuring ${ }^{11} \mathrm{C}-\mathrm{PIB} \mathrm{RI}$, the ${ }^{11} \mathrm{C}$-PIB uptake was three times lower in patients 
Table 3. Results analyzed according to amyloid fibril type

\begin{tabular}{lccc}
\hline & Type A $(\boldsymbol{n}=\mathbf{5})$ & Type B $(\boldsymbol{n}=\mathbf{5})$ & P value \\
\hline Age years (range) & $70(67-77)$ & $66(64-67)$ & 0.22 \\
Hypertension $n$ (\%) & $3(60 \%)$ & $2(40 \%)$ & 1.00 \\
Disease duration years (range) & $4(3-6)$ & $4(2-22)$ & 1.00 \\
NT-proBNP ng. ${ }^{-1}$ (range) & $220(146-341)$ & $711(134-3372)$ & 0.69 \\
Hs-Troponin T ng. ${ }^{-1}$ (range) & $14(12-16)$ & $31(24-169)$ & 0.11 \\
IVSD mm (range) & $14(12-16)$ & $13(12-14)$ & 0.40 \\
LA volume ml (range) & $48(35-59)$ & $63(44-89)$ & 0.18 \\
LVEF (range) & $0.56(0.52-0.64)$ & $19.2(11.7-21.9)$ & 0.17 \\
LV global strain-\% (range) & $19.2(15.9-24.0)$ & $0.05 \%)$ & 0.55 \\
DPD uptake $n$ (\%) & $5(100 \%)$ & $0.138(0.083-0.179)$ & $<0.01$ \\
Global LV ${ }^{11}$ C-PIB RI (range) & $0.042(0.032-0.046)$ & & $<0.01$
\end{tabular}

IVSD End-diastolic interventricular septum thickness; $L A$ left atrium; $L V$ left ventricle; $L V E F$ left ventricular ejection fraction; $R I$ retention index

Values are presented as median (min - max) or number (\%). Type A fibrils contain a mix of full-length and truncated ATTR and type B fibrils contain full-length fibrils only
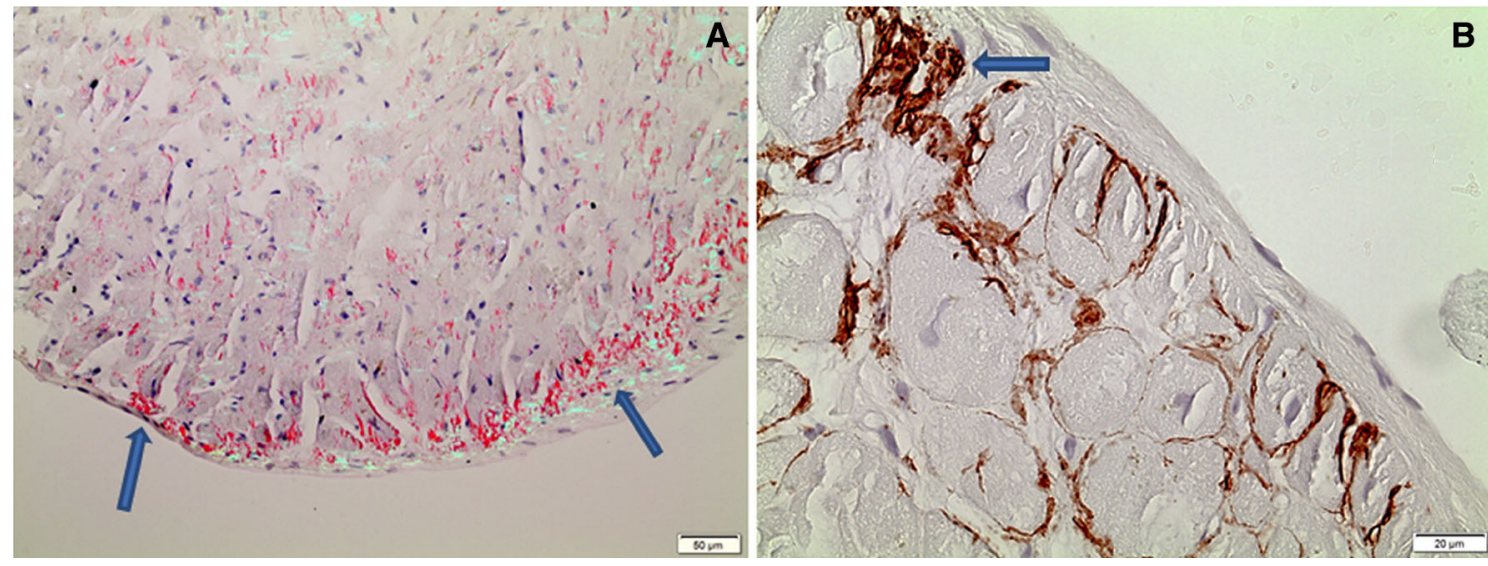

Figure 3. Endomyocardial biopsy stained with Congo red and visualized in polarized light between crossed polars (A). A considerable amount of amyloid is present subendocardially (arrows) but also between cardiomyocytes. In $\mathbf{B}$, amyloid is immunolabeled with an antibody against TTR. Bar in A $50 \mu \mathrm{m}$ and in B $20 \mu \mathrm{m}$.

with type A fibrils compared to those with type B fibrils (Table 3, mean $0.040 \pm 0.006$ vs $0.129 \pm 0.041$, $P=0.001)$. The pattern of ${ }^{11} \mathrm{C}-\mathrm{PIB}$ uptake differed between the two groups. In the type A group, the uptake was relatively homogenous in all patients, but in the type B group, uptake was patchy and unevenly distributed throughout the myocardium in four out of the five patients (Figure 2).

\section{Endomyocardial Biopsies}

One patient (no 9) in the study population had seven years prior to the study undergone an EMB, which was positive for amyloid. Only one (no 3 ) of the remaining four patients with type $\mathrm{B}$ amyloid agreed to the procedure. In that patient the biopsy showed rich subendocardial as well as myocardial amyloid deposits, appearing in part intracellularly (Figure 3).

\section{DISCUSSION}

We have previously shown that ATTR Val30Met fibrils are of two different variants of which one consists of full-length TTR (type B) and the other of a mixture of full-length TTR, and TTR fragments starting around position 50 (type A). ${ }^{7}$ Type A and type $\mathrm{B}$ deposits have different histological appearance that includes fibril morphology, tinctorial properties with Congo red as well 
as distribution in the cardiac muscle. It is therefore interesting that ${ }^{11} \mathrm{C}$-PIB seems to bind much stronger to type B than to type A fibrils. Therefore, the quantitative aspects of ${ }^{11} \mathrm{C}$-PIB binding to ATTR amyloid might depend on fibril type, rather than amyloid burden. In addition to different binding properties of type $\mathrm{A}$ and $\mathrm{B}$ fibrils, it is not possible to exclude factors that would minimize binding access such as fibrotic encapsulation of amyloid deposits and reduced capillary patency in the proximity of amyloid.

This reasoning holds true also for DPD. Absent DPD uptake in type B hearts suggests that DPD either does not have a binding site on type B fibrils or binding occurs related to a secondary phenomenon that is not present in cardiac type $\mathrm{B}$. The exact binding site for DPD is not known, but since DPD is widely used for imaging of bone mineralization, the most likely binding is associated with microcalcification. A case study using ${ }^{18} \mathrm{~F}$-sodium fluoride and PET, which is a specific marker of cardiac microcalcification, ${ }^{22}$ was recently shown to identify TTR amyloid, ${ }^{23}$ thus supporting that microcalcification occurs in TTR. The current study indicates that not only radiolabeled ligands but also therapeutic drugs can be expected to exert different binding properties in the context of amyloid fibril subtype.

A positive DPD scintigraphy is strongly associated with future development of amyloid cardiomyopathy. DPD scintigraphy in our study was positive only in patients with type A fibrils. ${ }^{11} \mathrm{C}$-PIB retention was three times higher in patients with type B fibril, compared to the group with type A fibrils. This suggests that the magnitude of ${ }^{11} \mathrm{C}$-PIB retention does not necessarily imply cardiomyopatic development. Echocardiographic abnormalities in patients with type B fibrils might in part be ascribed by other factors. In our study, $2 / 3$ type $B$ patients with increased LV wall thickness had long standing renal insufficiency and hypertension. Our study further suggests that the level and pattern of ${ }^{11} \mathrm{C}$-PIB and DPD retention are strongly associated with fibrillar type, indicating that the combination of ${ }^{11} \mathrm{C}$-PIB PET and DPD scintigraphy might be used for accurate subclassification of the ATTR fibril type.

The ${ }^{11} \mathrm{C}$-PIB retention that we measured was compared to a small group of healthy volunteers with no signs or history of cardiac pathology. Although these individuals most likely have no cardiac amyloid depositions, the PIB RI was not equal to zero. This is related to the low resolution of the PET scanner $(5-6 \mathrm{~mm})$, causing spill-over of activity from the blood pool into the myocardial region of interest. Visually, as shown in Figure 1, the absence of retention in the healthy volunteers is obvious in comparison to all patients. False positive results have so far not been reported, but larger cohorts are still needed to calculate the true sensitivity and specificity of ${ }^{11} \mathrm{C}$-PIB in cardiac amyloidosis.

The presence of amyloid depositions in endomyocardial biopsies from patients with positive DPD scintigraphy has been used to secure the diagnosis of cardiac amyloidosis. ${ }^{24}$ Biopsy proof of absence of amyloid deposition in cardiac tissues from patients with negative DPD scintigraphy has not been provided. It is, however, well known that amyloid deposition can be detected in tissues from well-functioning and macroscopically normal appearing organs such as the GI-tract or adipose tissue. ${ }^{11} \mathrm{C}$-PIB PET has been used extensively in detection of brain amyloid since 2002. The incidental finding of increased ${ }^{11} \mathrm{C}$-PIB uptake in cortical brain areas of clinically non-demented subjects initially caused concern about the specificity of ${ }^{11} \mathrm{C}$-PIB for diagnosis of Alzheimer's disease. However, the majority of these subjects developed clinically detectable dementia within a follow-up period of a few years. The finding of abnormal PIB uptake in the brain of patients with mild cognitive dysfunction is now considered prodromal Alzheimer's disease and is a validated inclusion criteria in drug trials. ${ }^{25}$ Thus, our findings of positive ${ }^{11} \mathrm{C}$-PIBzz PET examination of echocardiographically normal appearing hearts are not unexpected and could prove clinically valuable. Early detection of the disease before irreversible cardiac or neurological damage has occurred is important and the long-term outcome of novel pharmacological therapies will be depending on the severity of organ damage at the time of diagnosis. Sampling errors using biopsies from any organ are not uncommon and direct cardiac biopsies are often avoided until the clinical presentation is manifest. Based on the preliminary results of our study, cardiac ${ }^{11}$ C-PIB PET might be a sensitive marker of early systemic ATTR amyloidosis. It would therefore be interesting to utilize ${ }^{11} \mathrm{C}$-PIB PET in diagnosis of disease onset in mutation carriers with follow-up.

\section{Limitations}

The study was performed on a small number of patients and controls. In addition, EMB was only performed on two patients, which, however, were positive. A full compartmental analysis of PIB-PET data was not performed, and might have contributed additional insights into binding of PIB to myocardial amyloid. In brain studies of Alzheimer's patients, kinetics were best described using models of reversible binding but did not add to the clinical interpretation.

Due to the logistical reasons and high prevalence of pacemaker carriers, cardiac MRI is not performed routinely at our center and none of the patients underwent such investigation in conjunction with the study. 


\section{Summary}

The present study evaluated two different aspects of molecular imaging in a well-characterized cohort of patients with a certain diagnosis of ATTR V30M. All patients had myocardial PIB uptake on PET scan, regardless of fibril type or DPD uptake, but with significantly higher PIB retention in patients with fulllength fibril type, generally associated with less cardiac involvement. Myocardial DPD uptake, on the other hand, was only elevated in patients with the fragmented fibril type.

Since ${ }^{11}$ C-PIB RI appears influenced by factors other than mere amyloid burden, its use for amyloid quantification in clinical practice might be limited. ${ }^{11} \mathrm{C}$ PIB PET and perfusion studies may be useful to further study amyloid depositions in vivo and their impact on tissue metabolism. It would be interesting to follow disease progression and also to measure the effect of medical treatment on cardiac amyloid deposition and tissue metabolism. This is attractive since it can be applied to pacemaker-treated patients, where cardiac magnetic resonance imaging is unsuitable.

\section{NEW KNOWLEDGE GAINED}

C-PIB PET appears to be a very sensitive method for detecting cardiac ATTR amyloid deposits even in patients with primarily neurological disease ${ }^{11}$ and normal echocardiographic appearance. DPD scintigraphy appears to only detect ATTR deposits containing fragmented TTR, which previous studies have shown to cause more severe clinical heart disease.

\section{Disclosure} disclose.

None of the authors have any conflicts of interest to

\section{Open Access}

This article is distributed under the terms of the Creative Commons Attribution 4.0 International License (http://creativecommons.org/licenses/by/4.0/), which permits unrestricted use, distribution, and reproduction in any medium, provided you give appropriate credit to the original author(s) and the source, provide a link to the Creative Commons license, and indicate if changes were made.

\section{References}

1. Falk RH, Dubrey SW. Amyloid heart disease. Prog Cardiovasc Dis. 2010;52:347-61.

2. Rowczenio DM, Noor I, Gillmore JD, Lachmann HJ, Whelan C, Hawkins PN, et al. Online registry for mutations in hereditary amyloidosis including nomenclature recommendations. Hum Mutat. 2014;35:E2403-12.

3. Rapezzi C, Quarta CC, Riva L, Longhi S, Gallelli I, Lorenzini M, et al. Transthyretin-related amyloidoses and the heart: A clinical overview. Nat Rev Cardiol. 2010;7:398-408.

4. Ihse E, Ybo A, Suhr O, Lindqvist P, Backman C, Westermark P. Amyloid fibril composition is related to the phenotype of hereditary transthyretin V30M amyloidosis. J Pathol. 2008;216:253-61.

5. Arvidsson S, Pilebro B, Westermark P, Lindqvist $P$, Suhr OB. Amyloid cardiomyopathy in hereditary transthyretin V30M amyloidosis-impact of sex and amyloid fibril composition. PLoS One. 2015;10:e0143456.

6. Koike H, Misu K, Sugiura M, Iijima M, Mori K, Yamamoto M, et al. Pathology of early- vs late-onset TTR Met30 familial amyloid polyneuropathy. Neurology. 2004;63:129-38.

7. Bergstrom J, Gustavsson A, Hellman U, Sletten K, Murphy CL, Weiss DT, et al. Amyloid deposits in transthyretin-derived amyloidosis: Cleaved transthyretin is associated with distinct amyloid morphology. J Pathol. 2005;206:224-32.

8. Ihse E, Rapezzi C, Merlini G, Benson MD, Ando Y, Suhr OB, et al. Amyloid fibrils containing fragmented ATTR may be the standard fibril composition in ATTR amyloidosis. Amyloid. 2013;20:142-50.

9. Ericzon BG, Wilczek HE, Larsson M, Wijayatunga P, Stangou A, Pena JR, et al. Liver transplantation for hereditary transthyretin amyloidosis: After 20 years still the best therapeutic alternative? Transplantation. 2015;99:1847-54.

10. Rapezzi C, Quarta CC, Guidalotti PL, Longhi S, Pettinato C, Leone $\mathrm{O}$, et al. Usefulness and limitations of ${ }^{99 \mathrm{~m}} \mathrm{Tc}-3,3$-diphosphono-1,2-propanodicarboxylic acid scintigraphy in the aetiological diagnosis of amyloidotic cardiomyopathy. Eur J Nucl Med Mol Imaging. 2011;38:470-8.

11. Nordberg A, Carter SF, Rinne J, Drzezga A, Brooks DJ, Vandenberghe R, et al. A European multicentre PET study of fibrillar amyloid in Alzheimer's disease. Eur J Nucl Med Mol Imaging. 2013;40:104-14.

12. Antoni G, Lubberink M, Estrada S, Axelsson J, Carlson K, Lindsjo $\mathrm{L}$, et al. In vivo visualization of amyloid deposits in the heart with 11C-PIB and PET. J Nucl Med. 2013;54:213-20.

13. Lee SP, Lee ES, Choi H, Im HJ, Koh Y, Lee MH, et al. (11)CPittsburgh B PET imaging in cardiac amyloidosis. JACC Cardiovasc Imaging. 2015;8:50-9.

14. Dorbala S, Vangala D, Semer J, Strader C, Bruyere JR Jr, Di Carli MF, et al. Imaging cardiac amyloidosis: A pilot study using (1)(8)F-florbetapir positron emission tomography. Eur J Nucl Med Mol Imaging. 2014;41:1652-62.

15. Park MA, Padera RF, Belanger A, Dubey S, Hwang DH, Veeranna $\mathrm{V}$, et al. 18F-florbetapir binds specifically to myocardial light chain and transthyretin amyloid deposits: autoradiography study. Circ Cardiovasc Imaging. 2015;8:e02954.

16. Kero T, Lindsjo L, Sorensen J, Lubberink M. Accurate analysis and visualization of cardiac C-PIB uptake in amyloidosis with semiautomatic software. J Nucl Cardiol 2015.

17. Quinones MA, Otto CM, Stoddard M, Waggoner A, Zoghbi WA, Doppler Quantification Task Force of the N, et al. Recommendations for quantification of Doppler echocardiography: A report from the Doppler Quantification Task Force of the Nomenclature and Standards Committee of the American Society of Echocardiography. J Am Soc Echocardiogr. 2002;15:167-84.

18. Lang RM, Bierig M, Devereux RB, Flachskampf FA, Foster E, Pellikka PA, et al. Recommendations for chamber quantification. Eur J Echocardiogr. 2006;7:79-108.

19. Delgado V, Mollema SA, Ypenburg C, Tops LF, van der Wall EE, Schalij MJ, et al. Relation between global left ventricular 
longitudinal strain assessed with novel automated function imaging and biplane left ventricular ejection fraction in patients with coronary artery disease. J Am Soc Echocardiogr. 2008;21:1244-50.

20. Yingchoncharoen T, Agarwal S, Popovic ZB, Marwick TH. Normal ranges of left ventricular strain: a meta-analysis. J Am Soc Echocardiogr. 2013;26:185-91.

21. Ericzon BG, Larsson M, Wilczek HE. Domino liver transplantation: risks and benefits. Transplant Proc. 2008;40:1130-1.

22. Irkle A, Vesey AT, Lewis DY, Skepper JN, Bird JL, Dweck MR, et al. Identifying active vascular microcalcification by $(18) \mathrm{F}$ sodium fluoride positron emission tomography. Nat Commun 2015;6:7495.
23. Van Der Gucht A, Galat A, Rosso J, Guellich A, Garot J, Bodez D et al. [18F]-NaF PET/CT imaging in cardiac amyloidosis. J Nucl Cardiol 2015

24. Rapezzi C, Quarta CC, Guidalotti PL, Pettinato C, Fanti S, Leone $\mathrm{O}$, et al. Role of $(99 \mathrm{~m}) \mathrm{Tc}-\mathrm{DPD}$ scintigraphy in diagnosis and prognosis of hereditary transthyretin-related cardiac amyloidosis. JACC Cardiovasc Imaging. 2011;4:659-70.

25. Ma Y, Zhang S, Li J, Zheng DM, Guo Y, Feng J, et al. Predictive accuracy of amyloid imaging for progression from mild cognitive impairment to Alzheimer disease with different lengths of followup: A meta-analysis. Medicine 2014;93:e150. 